The aponeurosis of the external oblique muscle is now hrought together by a continuous suture of fine silk, and the skin wound by interrupted sutures of silkworm gut. Then a small collodion antiseptic dressing is applied and the parts left untouched for five or six days, when the dressing is removed and the superficial stitches cut out and the wound redressed.

The child need not be kept in bed longer than ten days, after which period it may be allowed to run about, provided that sound healing has taken place. There is no necessity for such a patient to wear a truss subsequent to the operation, unless the parts previously had been much dilated by a large hernia. In this instance it is well that a truss should be applied for about a year as a precaution. The results of these operations are most satisfactory, and it is doubtful whether a promise can really be made of a lasting cure, save in the congenital inguinal herniæ of children.

\section{IRREDUCIBLE HERNiA.}

So far I have only dealt with reducible herniæ. A hernia which is in a condition of simple irreducibility, that is, one which is irreducible, but not strangulated, is not common in young children, but it does occur occasionally as the outcome of a congenital cæcal hernia on the right side, and more rarely owing to adherent omentum.

Both of these irreducible herniæ are perhaps best treated by operation, although if this for any reason is contra-indicated, then the application of a hinged-cup truss is to be advised.

When strangulation occurs in the congenital inguinal herniæ of childhood, it is seldom very acute - that is to say, the signs of intestinal obstruction are not well marked. Moreover, reduction is usually comparatively easy by taxis, or the hernia may become reduced either spontaneously or after the lower limbs and pelvis of the patient have been kept elevated for a few hours.

If these means fail, then herniotomy is necessary and should be performed together with a radical treatment of the sac.

\section{TWO CASES OF RESECTION OF THE LARGE INTESTINE, WITH RECOVERY.}

BY FREDERIC EVE, F.R.C.S.,

Surgeon to the London Hospital and to the Evelina Hospital for Sick Children.

These two cases are examples of the commonest causes for which the large intestine may require resection-namely, for strangulation and gangrene by hernia, and for carcinoma. They both illustrate the wisdom of postponing resection when obstruction exists until this has been relieved. Case II is published especially to draw attention to the merits of extraperitoneal resection and suture for artificial anus.

CASE I. Strangulated Left Inguinal Hernia: Herniotomy: Perforation of Transverse Colon: Resection of Bowel Five Weeks Later : Recovery.-T. T. aged 46, was admitted to the London Hospital on November $23 \mathrm{rd}$, 1896, with a large, strangulated, left serotal herma. The hernia had existed for twenty years, and he had worn a truss. On November 20 th the rupture the do the 22nd instant. The bowels had not acted sincethe rgth. On November ${ }_{23}^{2}$ rd herniotom tion of the transverse colon. This was deeply congested and perforated. the opening was too large and the condition of the bowel too unsatisfactory to admit of closing the opening by sutures. A glass tube (Paul's) was thin in the inguinal region. The pationt skin in the inguinal region. The patient recovered rom this operation without any troublesome complications. A portion of bowel covered with granulations protruded from an opening in the abdominal wall, and the whole of the faces were discharged through it. After allowing an interval of five weeks to elapse in order that the parts might consolidate and the inflammatory thickening disappear, the operation of resection was performed on January 2nd, r897. Anæsthetic, ether and A.C. E. mixture. An incision was made vertically upwards in the semilunar line from the small protrusion of the bowel. This divided all the structures down to the peritoneum. It was my intention to perform the resection entirely extraperitoneally, after the manner suggested by the late Mr. Greig Smith. ${ }^{2}$ But the peritoneum was found so thin and delicate that it would not permit of the necessary manipulation. This was doubtless due to stretching of the membrane from the proximity of the hernia. The peritoneal cavity was therefore opened, the proximal end of bowel secured, and the protruded portion Then separated by the division of the abdominal parietes around it. abdominal wound and the peritoneal cavity protected with sponges. The gut was resected, and the divided mesentery brought together with a running suture. The mucons membrane of the free ends of the intestine and muscular coats were united by about fifty Lembert sutures. The running suture through the mesentery was tied last Finally, a graft of omentum was fixed around the line of sutures, especially near the mesenteric attachment. The abdominal wound was closed and a small drain was left in it. This was removed in twenty-four hours. The operation lasted about two hours. The patient suffered considerably from shock after the operation, but was never sick, and the wound healed by first intention. He was fed by nutrient enemata until the fourth day, when champagne and milk were given by the mouth. On January 28 th he was

CASE II Carcinoma

CASE II. Carcinoma of Descending Colon: Colectomy: Recovery.-B. D., aged 47, was admitted to the London Hospital under the care of my
colleague, Dr. F. J. Smith, on Janualy $28 \mathrm{th}$, r898, suffering with chronic onstipation.

History.-Her illness commenced at the beginning of the summer or 897 with pain in the abdomen and vomiting. The vomiting occurred about wo hours after a meal, and the pain was relieved by it. Some months ago

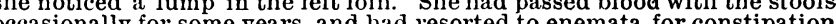
cer tonally some son for tho years. Lately tenesmus had in weight within the last six months. She was transferred to the surgicar the kidn was ary the kidney was a sausage-shaped tumour distinctly movable from side to side, and evidently situated in the descending colon. No enlargement of the liver existed. Th abdomen the descending colon was diagnosed. As a preparation for resection of that bel amid purgative (magnesize sulph.) was given, with the result vomited. Operation.-February roth, r898. Anæsthetic, ether. The tumour was. constricting carcinoma of the descending colon just below the splenic flexure. The tumour was brought out through the wound, together with some inches of bowel on each side of it, and was stitched to the parietes, the wound being partially closed. $\Lambda \mathrm{n}$ incision was then made into the bowel on the proximal side of the tumour, and a glass tube (Paul's) was tied in. It was not thought advisable to perform resection and suture of the intestine immediately, owing to the fact that the intestines were conserious an operation.

February 2ist. The portion of the bowel with the tumour protruding from the wound was cut away level with the skin. The incision was so planned that more of the concavity than of the convexity of the propruding knuckle was removed. in order to permit subsequently of easier approximation of the ends. In attempt was made to prevent formation of a spur by suturing together the mesenteric borders of the ends. Microscopic examination of the tumour sliowed it to be a typical columnarinflammatory effusions around the wound to become absorbed.

On March 25 th. under ether, an incision through the skin and parietes was made around the colotomy wound, and this was continued above and below the artificial anus for a considerable distance. These incisions were carried down to the peritoneum, which was then separated from the parietes for a distance of $x^{2}$ inch to 2 inches all round the bowel. The collar of skin attached to the divided ends of the bowel was removed, and the latter were refreshed, except at the united mesenteric borders. The ends of the bowel were then brought together by two rows of sutures, oneuniting the mucous membrane, and the other the peritoneum and muscular coat. The bowel with the peritoneum was then pushed inwards, and the parietes united with silkworm gut, the wound being completely closed with the exception of a small gauze drain. Food by the mouth was given on the fourth day.

On April 3rd, nine days after the operation, a small fistula formed, and a slight fæcal discharge appeared on the dressings. Subsequently the wound was washed out with $x$ in 4,000 solution perchloride of mercury whenever the dressings were changed, but there was no further discharge of fieces. The sinus was quite closed on April 2oth. The patients was then allowed to get up. She is now (May, 1899) perfectly well, with no. symptom of recurrence.

The propriety of draining the perforated intestine at the first operation in Case $I$, instead of attempting an immediate resection, is scarcely open to doubt. This remark applies to the present day, when Murphy's button is so much used, nearly as forcibly as it did in 1896 , when the case related occurred : and it would apply more strongly to the large intestine than to the small. The introduction of Murphy's button has not in my opinion affected the justice of the conclusion expressed by Mr. Greig Smith as follows: "I have come to the conclusion that to resect living intestine during obstruction is surgically unsound; and I doubt if any improvement in methods will ever make the operation justifiable. The shock and the illness which follow resection of bowel are too great to put on a patient suffering from intestinal obstruction." It has occurred to me that a perforation or other limited lesion of intestine, not suitable for suture, might be treated by an invagination of a portion of the circumference of the bowel, the invaginated part being retained in position by sutures but I have not had a favourable opportunity of putting this to the test. Before performing resection $I$ considered whether I would suture or use a Murphy's button, and came to the conclusion that for the large intestine, at any rate, the former was the safer.

In regard to Case in the same remarks concerning immediate resection at the first operation apply, although the obstruction 
was not ncarly so acute as in the first case related. An amount of partial obstruction had, however, existed for a considerable period; and this during the day or two preceding the operation had become complete.

I would strongly advocate the method of extraperitoneal suture as the safest, and that which is most free from anxiety. It is, however, not easy to strip up the peritoneum from the parietes to a sufficient extent without tearing it ; but slight rents may be closed with a continuous suture of fine silk. The suturing of the intestine is also more difficult, owing to the position of the ends of the intestine. It was especially so in the case reported, because the walls of the bowel were sodden and very friable; and $I$ had at the time considerable doubt as to whether the sutures would hold. They did not, in fact, do so completely, although the ultimate result was not affected.

REFERENCE.

1 Abdominal Surgery, vol. ii, p. 634

\section{A CASE OF ARRESTED DEVELOPMENT OF THE THIRD AND FOURTH RIBS.}

By ALFRED G. LEVY, M.D.LoND.

I FoUND this abnormality in a somewhat poorly-developed boy of 13 years of age. The general features of the case are as follows :

The front of the chest on the right side (Fig. I) presents a striking depression over the position corresponding normally

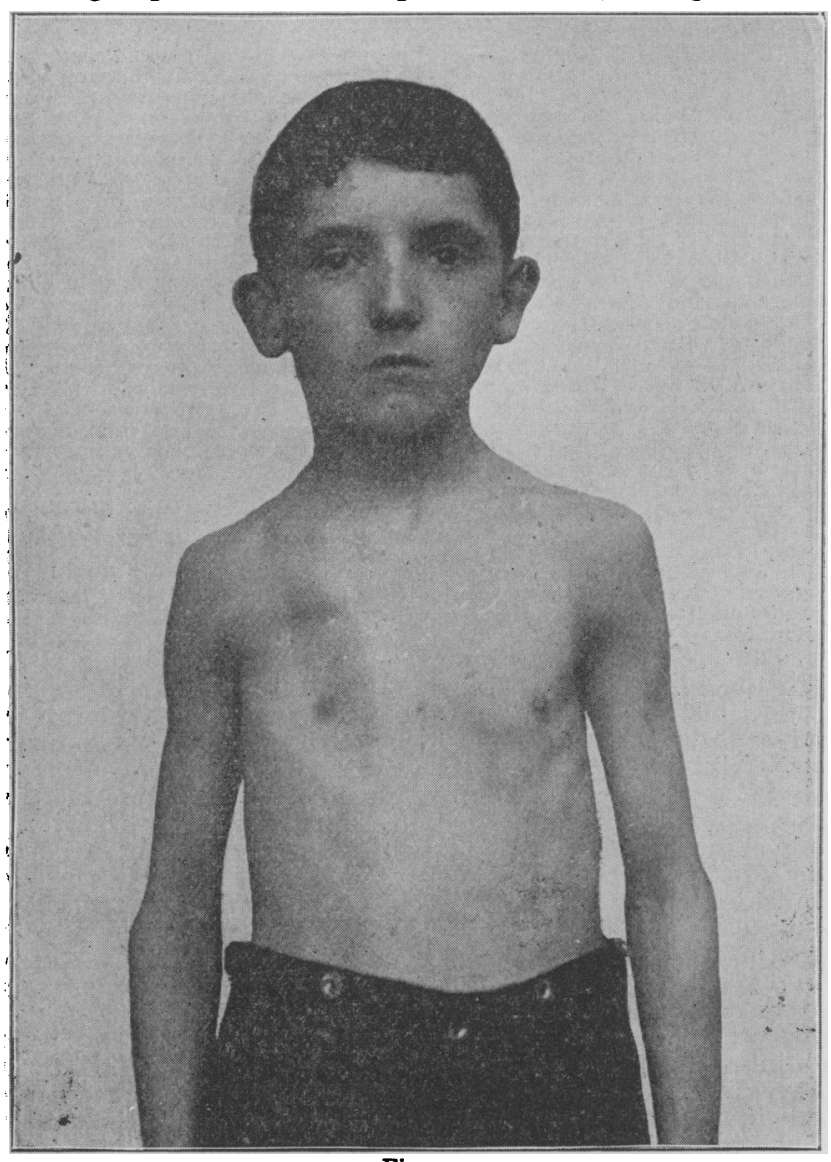

Fig. 1.

to that of the third and fourth ribs, and this appearance is accentuated by the exaggerated development of the clavicular portion of the pectoralis major, and of a small neighbouring portion arising from the first rib cartilage. There is no further trace of pectoral muscle, either major or minor.
The third rib cartilage is a little stump about half an inch in length, but the fourth is a narrow curved strip two-and-ahalf inches long lying close along the upper border of the nifth cartilage. The end of the curtailed third rib may be obscurely felt in the axilla just under the margin of the pectoral muscle and lying close to the second rib while the attenuated termination of the fourth rib is found a little further back in the axilla. The skiagraph, made

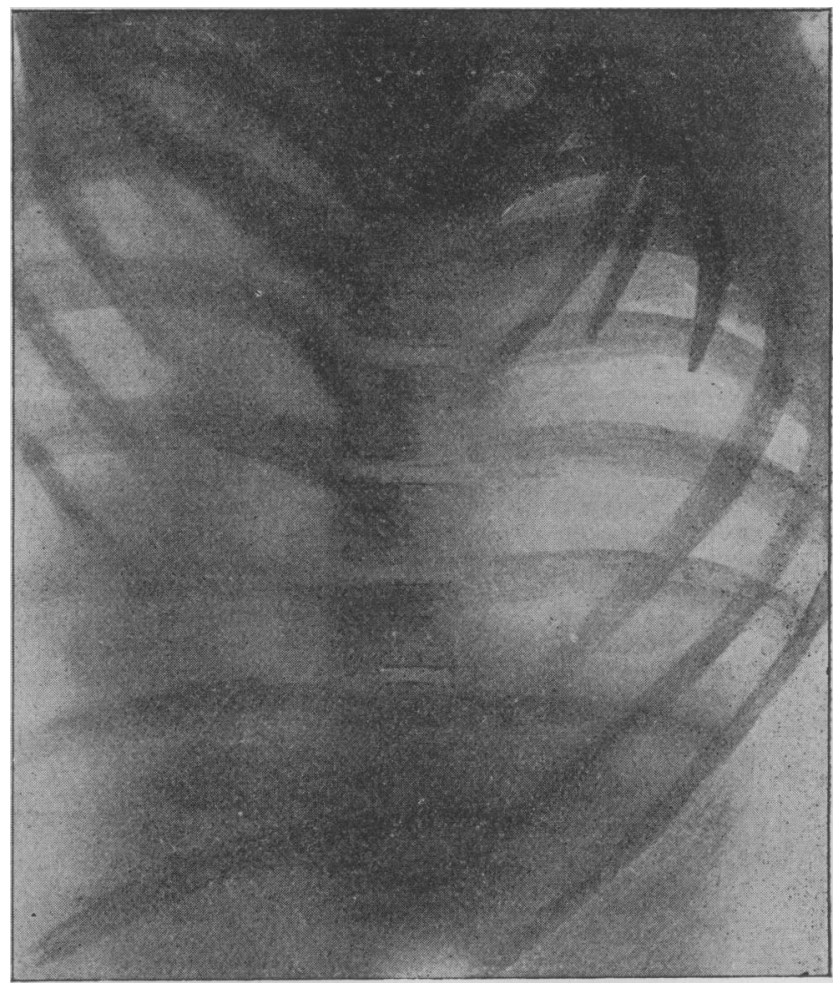

Fig. 2.

for me by Messrs. Coxeter and Son (Fig. 2), was taken with the boy lying in the prone position, so that it represents a front view of the thorax, and the tapering ends of the malformed ribs are seen in the skiagraph.

Naturally some spinal deformity results. There is a well marked dorsal curvature with little compensatory curve either in the lumbar or the cervical regions; this is the usual type of curvature found in cases of collapse of the ribs, such as may be found following an empyema, but whereas in the latter case the convexity of the curvature would be directed towards the healthy side, in the present instance the spinal column is deflected towards the malformed side, as perhaps might be expected from the deficiency of muscular and bony support involved therein. Judging from the breadth of the intercostal spaces on the two sides of the chest, there does not appear to be much rotation of the vertebræ, but it is probable that matters have been considerably modified by a systematic course of gymnastics which the patient underwent about a year ago with markedly beneficial results.

Abnormal conditions of the chest such as I have just described are decidedly rare. Now and again a case of complete absence of some of the ribs, usually the asternal ones, is reported, the condition being sometimes associated with imperfect development or absence of corresponding vertebræ; but it would certainly appear that ribs tend more to vary in the direction of complete absence than in that of mere incompleteness of development. The first rib is partly responsible for examples of the last-named deformity, but L. E. Hertslet states that only 5 cases of rudimentary first ribs are recorded in Great Britain, and $\mathrm{Helm}^{2}$ can find only 16 in the general anatomical literature of the past twenty-five years. Both of the above-mentioned authorities record cases, as also does 